\title{
Lateral transport in disordered layered media studied by the transfer-matrix method
}

\author{
Man-Chung Chan and Zhao-Qing Zhang \\ Department of Physics, Hong Kong University of Science and Technology, Clear Water Bay, Kowloon, Hong Kong
}

(Received 4 December 1996)

\begin{abstract}
In the theory of products of random matrices, the Lyapunov exponents are normally obtained by employing the Gram-Schmidt orthonormalization procedure together with the transfer matrices. The divergence of the volume spanned by various orthonormalized vectors determines the exponents. However, the vector nature of these orthonormalized vectors has been largely ignored. We show that the vector which corresponds to the localization length exponent actually contains the statistical information on the relative layer occupation probability. This is the key quantity used to describe the lateral transport in disordered layered media.
\end{abstract} [S0163-1829(97)05725-1]

The transfer matrix (TM), which consists of a product of random matrices, has become the standard method in the study of various physical problems. ${ }^{1}$ Typical examples are the dynamical properties of classical chaotic systems and the transport properties of disordered systems., ${ }^{2,3}$ One of the main physical quantities interested in such studies is the Lyapunov spectrum. In the numerical calculations of Lyapunov exponents, a Gram-Schmidt orthonormalization procedure is normally employed to separate various exponents. While the divergence of the norms of various orthogonal vectors determines the exponents, the vector nature of these vectors has been largely ignored in the past. In the study of transport properties of disordered systems, the only measurable exponent is the exponent which has the smallest absolute value. This is due to a relation between the transfer matrix and the transmission matrix, which relates the slowest growth length to the localization length of the system. ${ }^{4}$

However, in the study of the lateral transport of disordered layered systems such as the Earth's subsurface and random superlattices with isotropic inhomogeneities, ${ }^{5}$ it has been shown that one of the most important physical quantities characterizing such systems is the nonuniform distribution of layer occupation probability. ${ }^{6,7}$ For a system of finite cross section, i.e., a multichannel problem, it has also been shown both analytically and numerically that there exists an equilibration length, beyond which the system reaches an asymptotic regime and the relative layer occupation probability becomes stationary. Like the Lyapunov exponents, this stationary distribution is the global property of the system, independent of initial condition and configuration. There are other similar multimode conversion problems. ${ }^{8,9}$ For instance, in the case of elastic wave propagation through layered media, the interconversion of shear and compressional waves due to scatterings gives rise to a fixed probability density of the ratio of two components in the asymptotic regime after a certain equilibration length. ${ }^{9}$ The stationary nonuniform layer distribution in disordered layered systems dictates one-dimensional-like transport behaviors of such systems. ${ }^{7}$ In order to obtain this information, we normally have to calculate the transmission matrix for different incoming and outgoing channels. This can be done by performing the recursive Green's function (RGF) calculations. ${ }^{3,10}$ Unlike the TM, the RGF method requires matrix inversions and can only produce the exponent which is the inverse of the localization length. Other exponents have to be obtained in an indirect way. ${ }^{7}$

In this work, we show that the vector nature of the orthonormalized vectors obtained from the Gram-Schmidt procedure in the TM method actually contain the statistical information of the layer occupation probability. In the asymptotic regime, the vector which provides this information is the one that corresponds to the exponent with the smallest absolute value. Thus, by using the TM method, we can obtain not only all the Lyapunov exponents, but also the statistics about the relative layer occupation probability. The results of this study can be generalized to other physical problems involving multimode interconversion.

We use a tight-binding Hamiltonian for electronic system to describe a disordered layered medium, ${ }^{5-7}$

$$
H=\sum_{\alpha} \varepsilon_{\alpha}|\alpha\rangle\left\langle\alpha\left|+\sum_{[\alpha, \beta]} t\right| \alpha\right\rangle\langle\beta|,
$$

where $t$ is the hopping matrix element, $[\alpha, \beta]$ denotes the nearest-neighbor sites $\alpha$ and $\beta$ in the lattice. The random on-site energy $\varepsilon_{\alpha}$ consists of two parts,

$$
\varepsilon_{\alpha}=\overline{\varepsilon_{z}}+\gamma_{\alpha},
$$

where $\bar{\varepsilon}_{z}$ describes the layer energy which is a constant for all $x$ and $y$ for each fixed layer $z \cdot \gamma_{\alpha}$ is a random number that varies independently from site to site with a flat probability distribution of width $W$, which describes the isotropic inhomogeneity of the system. For simplicity, we assume here both $t$ and lattice constant to be unity. In a disordered layered medium such as random superlattice (or the Earth's subsurface), the layer energy $\bar{\varepsilon}_{z}$ in each layer is also random. If we use $W_{1}$ to denote the randomness of the layer energy, the ratio $\delta \equiv W_{1} / W$ describes the degree of anisotropy of the system. If $\delta=0$, the system is a $d$-dimensional isotropic system. If $\delta=\infty$, the system is a one-dimensional layered medium. Here, we consider only two-dimensional systems ( $d$ $=2$ ) with $z$ and $x$ as the layering and lateral directions, respectively. For a given layer configuration in a strip geometry, if electrons are injected laterally from one side of the sample, it has been found that the relative probability of electrons in each layer at the output surface is nonuniform. It 
has also been shown that there exists an equilibration length beyond which the relative layer occupation probability becomes stationary. ${ }^{6,7}$

In the anisotropic limit of large $\delta$, the equilibration length diverges and the layer distribution becomes singular. These interesting phenomena actually dictate the one-dimensionallike transport behavior of such systems and make them different from the anisotropic hopping model (AHM), where the hopping matrix $t$ in Eq. (1) is anisotropic but the site energy is isotropically random, i.e., $\overline{\varepsilon_{z}}=0$ in Eq. (2). ${ }^{11}$ In the AHM, the layer occupation probability is always uniform, independent of the anisotropy. This property ensures that the AHM belongs to the same universality class as the isotropic random systems. Therefore, the key quantity in the Hamiltonian, Eq. (1), is the relative layer occupation probability. This quantity has been studied previously by using the RGF in channel space. ${ }^{6,7}$ In order to compare the results obtained from the RGF and the TM methods, we first calculate the RGF in real space using the method outlined in Ref. 3.

Consider a strip geometry of $M$ layers and length $L$ with a given set of layer energy $\bar{\varepsilon}_{z}, z=1,2, \ldots, M$. If we inject electrons from the $i$ th layer at the input surface $x=1$, the amplitudes of the wave function in the $j$ th layer at the output surface $x=L$ can be described by the Green's function $\left[\mathbf{G}_{L, 1}\right]_{j, i}$, which is an $M \times M$ matrix. The Green's function $\mathbf{G}_{L, 1}$ can be obtained numerically by iterating the following equations from $n=1$ to $L-1$ :

$$
\begin{gathered}
\mathbf{G}_{n+1,1}=\mathbf{G}_{n+1, n+1} \mathbf{G}_{n, 1}, \\
\mathbf{G}_{n+1, n+1}=\left[E \mathbf{I}-\mathbf{H}_{n+1}-\mathbf{G}_{n, n}\right]^{-1},
\end{gathered}
$$

with $\mathbf{G}_{1,1}=\left[E \mathbf{I}-\mathbf{H}_{1}\right]^{-1}$. Here $E$ is the energy of the electron, $\mathbf{I}$ is the $M \times M$ identity matrix, and $\mathbf{H}_{n}$ is the isolated Hamiltonian matrix at $x=n$ which has the form

$$
\mathbf{H}_{n}=\left(\begin{array}{cccccc}
\varepsilon_{n, 1} & 1 & 0 & \cdots & 0 & 1 \\
1 & \varepsilon_{n, 2} & 1 & \cdots & 0 & 0 \\
0 & 1 & \varepsilon_{n, 3} & \cdots & 0 & 0 \\
\vdots & \vdots & \vdots & \ddots & \vdots & \vdots \\
0 & 0 & 0 & \cdots & \varepsilon_{n}, M-1 & 1 \\
1 & 0 & 0 & \cdots & 1 & \varepsilon_{n, M}
\end{array}\right),
$$

where a periodic boundary condition has been adopted across the strip and the subscript " $n, i$ " denotes the site position at $x=n$ and $z=i$. The localization length $\xi_{M}$ can be calculated from $\xi_{M}=-\lim _{L \rightarrow \infty} 2 L / \ln \operatorname{Tr}\left[\mathbf{G}_{L, 1}\right]$. It has been shown that, when $L$ is larger than some equilibration length $L_{\text {eq }}$, the slowest decay length dominates and $\mathbf{G}_{L, 1}$ has the asymptotic structure $\lim _{L \rightarrow \infty}\left[\mathbf{G}_{L, 1}\right]_{j, i}=a_{j}(L) g_{i}$, where $g_{i}$ is the corresponding eigenvector of $\lim _{L \rightarrow \infty}\left[\mathbf{G}_{L, 1}^{+}\right]\left[\mathbf{G}_{L, 1}\right] .{ }^{7}$ The $L$-independent eigenvector $g_{i}$ depends only on the site energy configuration before the equilibration length $L_{\text {eq }}$ and, therefore, is sample dependent. On the contrary, due to scatterings, $a_{j}(L)$ rotates in the $M$-dimensional space in a random way as $L$ varies. Albeit local fluctuations, $a_{j}(L)$ decays exponentially with the decay length $\xi_{M}$. After normalization, the relative occupation probability $p_{j}$ in each layer $j$ at the output surface becomes $\vec{a}_{j}^{2}(L)=a_{j}^{2}(L) /\left[\sum_{k=1}^{M} a_{k}^{2}(L)\right]$. In the asymptotic regime, the averaged layer occupation probability, which can be defined as

$$
\left\langle\bar{a}_{j}^{2}(L)\right\rangle_{L, L_{\mathrm{eq}}}=\frac{1}{L-L_{\mathrm{eq}}+1} \sum_{x=L_{\mathrm{eq}}}^{L} \vec{a}_{j}^{2}(x),
$$

becomes stationary in the large $L$ limit.

In the TM method, if we denote the wave function at $x$ $=n$ and $z=i$ by $\varphi_{n, i}$ and the row vector $\mathbf{z}^{T}(n)$ as $\left(\varphi_{n, 1} \varphi_{n, 2} \cdots \varphi_{n, M} \varphi_{n-1,1} \varphi_{n-1,2} \cdots \varphi_{n-1, M}\right)$, the column vector $\mathbf{z}(n+1)$ is related to $\mathbf{z}(n)$ by

$$
\mathbf{z}(n+1)=\mathbf{M}(n) \mathbf{z}(n),
$$

where $\mathbf{M}(n)$ is a $2 M \times 2 M$ matrix of the form ${ }^{1}$

$$
\mathbf{M}(n)=\left(\begin{array}{cc}
E \mathbf{I}-\mathbf{H}_{n} & -\mathbf{I} \\
\mathbf{I} & \mathbf{O}
\end{array}\right),
$$

where $\mathbf{H}_{n}$ is given in Eq. (5). The TM, which relates the wave function at the two output surfaces to that at the two input surfaces becomes $\mathbf{z}_{k}(L)=\mathbf{P}_{L} \mathbf{z}_{k}(0)$, with $\mathbf{P}_{L}$ $=\prod_{i=1}^{L} \mathbf{M}(i)$. The subscript $k=1,2, \ldots, 2 M$ denotes $2 M$ independent initial conditions for the TM. From the Oseledec theorem, the sum of the first $p$ largest Lyapunov exponents is determined by the divergence of the volume spanned by $p$ vectors $\mathbf{z}(k), k=1,2, \ldots, p .{ }^{1}$ Since the TM is symplectic, the Lyapunov exponents come in pairs with opposite signs. We can write the $M$ positive exponents as $\lambda_{1}>\lambda_{2}>\cdots>\lambda_{M}$. Since all $\mathbf{z}(i)$ 's diverge with the largest exponent, the TM has a similar asymptotic structure $\left[\mathbf{P}_{L}\right]_{j, i}=b_{j}(L) f_{i}$ to that of the Green's function in Eq. (3) in the large $L$ limit. The vector $f_{i}$ is the eigenvector of the asymptotic matrix $\lim _{L \rightarrow \infty}\left[\mathbf{P}_{L}^{+}\right]\left[\mathbf{P}_{L}\right]$ that corresponds to the maximum exponent $\lambda_{1}$, which is also the growth length of $b_{j}(L)$, apart from some fluctuations. ${ }^{1}$ Since the $\lambda_{1}$ corresponds to the shortest decay length in the transmission matrix, both the vectors $f_{i}$ and $b_{j}(L)$ are not the measurable quantities in the lateral transport considered here. The interesting nonuniform layer distribution probability in the asymptotic regime should be described by the vectors that correspond to the exponent $\lambda_{M}$ in the TM. Since, in the numerical calculations, both the eigenvectors of the asymptotic TM and the Lyapunov exponents that correspond to the exponents $\lambda_{i}$ with $i>1$ are masked by the largest exponent $\lambda_{1}$, the Gram-Schmidt normalization procedure is normally employed to separate each exponent. ${ }^{1,3}$ Here we orthonormalize the $2 M$ vectors $\mathbf{z}_{k}(x)$ at each step $x$ by using the following procedure:

$$
\begin{gathered}
\hat{\mathbf{z}}_{1}=\frac{\mathbf{z}_{1}}{\left\|\mathbf{z}_{1}\right\|}, \\
\hat{\mathbf{z}}_{2}=\frac{\mathbf{z}_{2}-\left(\hat{\mathbf{z}}_{1}^{T} \cdot \mathbf{z}_{2}\right) \hat{\mathbf{z}}_{1}}{\left\|\mathbf{z}_{2}-\left(\hat{\mathbf{z}}_{1}^{T} \cdot \mathbf{z}_{2}\right) \hat{\mathbf{z}}_{1}\right\|}, \\
\vdots \\
\hat{\mathbf{z}}_{2 M}=\frac{\mathbf{z}_{2 M}-\sum_{i=1}^{2 M-1}\left(\hat{\mathbf{z}}_{i}^{T} \cdot \mathbf{z}_{2 M}\right) \hat{\mathbf{z}}_{i}}{\left\|\mathbf{z}_{2 M}-\sum_{i=1}^{2 M-1}\left(\hat{\mathbf{z}}_{i}^{T} \cdot \mathbf{z}_{2 M}\right) \hat{\mathbf{z}}_{i}\right\|},
\end{gathered}
$$

where the superscript ' $T$ " denotes row vectors. We have omitted the bracket " $(x)$ "' in the above equations. The above 


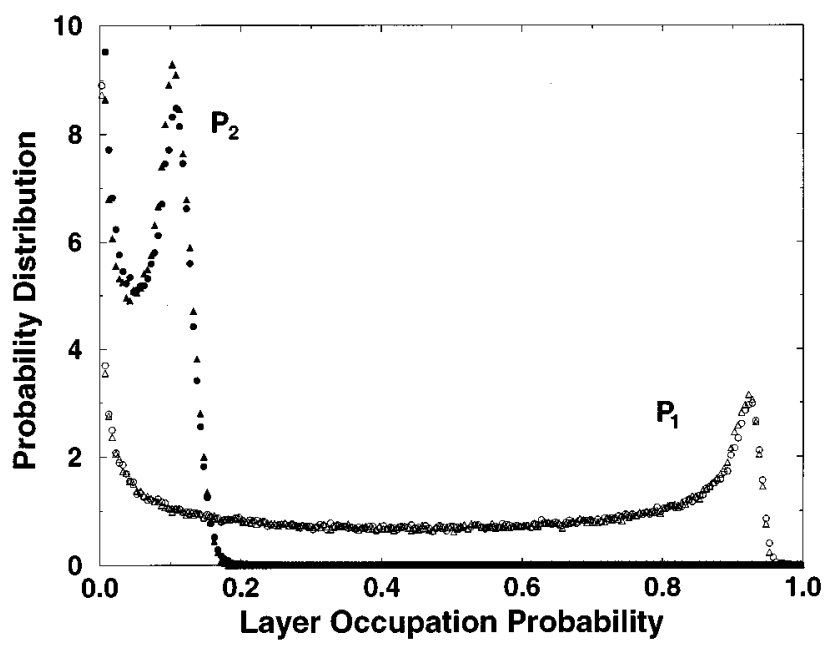

FIG. 1. For the three-layer system described in the text, the open and solid triangles denoted, respectively, the probability distributions of relative occupation probability in the first and second layer obtained from the recursive Green's function. The results obtained from the transfer-matrix method are shown by open and solid circles.

procedure is iterated from $x=1$ to $L$. Let $R_{k}(x)$ be the norms in the denominators of $\hat{\mathbf{z}}_{k}(x)$. Then the Lyapunov exponent $\lambda_{k}$ can be obtained from the large $L$ limit of $\sum_{n=1}^{L} \ln R_{k}(n) / L$. Since the exponent that corresponds to the localization length is $\lambda_{M}$, we would expect that the vector nature of the vector $\hat{\mathbf{z}}_{M}(x)$ should provide the statistical information about the layer occupation probability in the asymptotic regime when $x>L_{\mathrm{eq}}$. The rotation of $\hat{\mathbf{z}}_{M}(x)$ in $2 M$-dimensional space as $x$ varies is also due to isotropic scatterings. Since $\hat{\mathbf{z}}_{M}(x)$ contains the information about the wave function at both $x$ and $x-1$, we need to consider only half of the vector. Let us denote $\hat{z}_{i M}(x)$ as the $i$ th component of $\hat{\mathbf{z}}_{M}(x)$ and renormalize its upper-half vector to

$$
\tau_{i}(x) \equiv \frac{\hat{z}_{i M}(x)}{\sqrt{\sum_{j=1}^{M} \hat{z}_{j M}^{2}(x)}} .
$$

The occupation probability in the $i$ th layer becomes $p_{i}(x)$ $=\tau_{i}^{2}(x)$, where $i=1, \ldots, M$.

In the following, we show the results of layer occupation statistics obtained from both the RGF and TM methods in two different cases. In both cases, we have chosen the energy of electrons to be $E=0$ and the randomness to be $W=1$. First, we consider a three-layer system with layer energies $\overline{\varepsilon_{z}}=0,4$, and -0.5 for $z=1,2$, and 3 , respectively. The equilibration length in this case is found to be around 900. In the asympototic regime, the probability distributions obtained from the RGF in layer $z=1$ and $z=2$, i.e., $p_{1}=\vec{a}_{1}^{2}$ and $p_{2}=\bar{a}_{2}^{2}$, are shown in Fig. 1 by open and solid triangles, respectively. In the same figure, we also plot the distributions obtained from Eq. (10) of the TM by open and solid circles. In the case of TM, the statistics are taken from a single configuration with $x$ ranging from 1000 to 201000 . In the case of RGF, statistics of five configurations are taken with $x$ ranging from 1000 to 41000 . The excellent agreement between the results of the two methods demonstrates that the

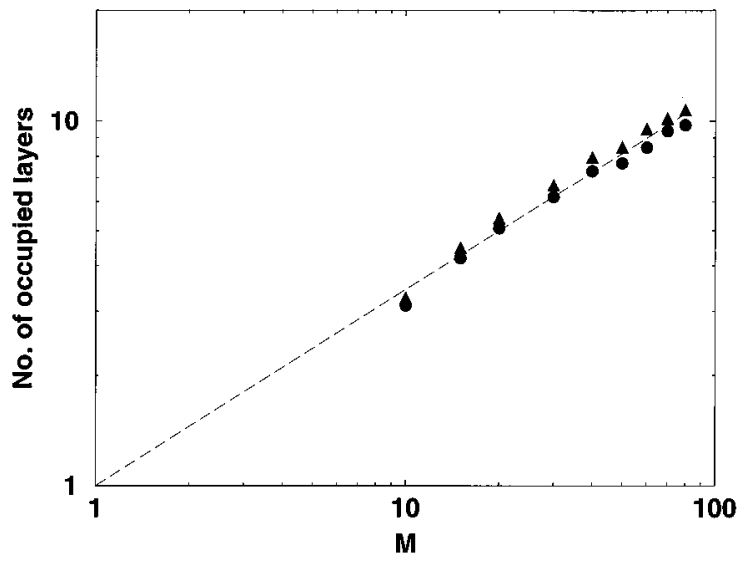

FIG. 2. The average number of occupied layers is plotted against the layer number in log-log scale for the case of $W_{1}=1$. The triangles and circles denote the data obtained from the RGF and $\mathrm{TM}$, respectively. The dotted line denotes the function $M^{0.54}$.

vector nature of $\hat{\mathbf{z}}_{M}(x)$ indeed provides the statistical information about the layer occupation probability. It should be mentioned that the fixed eigenvector $g_{i}$ of the asymptotic RGF, which depends on the energy configuration before the equilibration length, can also provide the layer occupation probability if the statistics are taken from a large number of configurations. From the results of 40000 configurations, we have obtained the same distributions as the ones shown in Fig. 1.

In order to show that Eq. (10) holds in general, we consider a case where the layer energies $\bar{\varepsilon}_{z}$ in Eq. (2) are also randomly distributed with a width $W_{1}=15$. If we keep $W$ $=1$, this represents a strongly anisotropic system with $\delta$ $=15$. The quantity we are interested is the average number of occupied layers $N_{\text {oc }}$, or inverse participation ratio, which is defined as ${ }^{6}$

$$
N_{\mathrm{oc}}(M)=\left\langle\frac{1}{\sum_{j=1}^{M} p_{j}^{2}(x)}\right\rangle,
$$

where $p_{j}(x)$ is the occupation probability in layer $j$ and denotes $\bar{a}_{j}^{2}(x)$ in the RGF and $\tau_{j}^{2}(x)$ in the TM. This function has been calculated in channel space previously and showed a dimensional crossover behavior of $N_{\mathrm{oc}}\left(M_{s}\right) \propto \sqrt{M_{s}}$, where $M_{s}$ denotes the number of propagating channels. If the system is isotropic, i.e., $W_{1}=0$, we have $N_{\text {oc }}(M)=M$, while for one-dimensional behavior, we expect that $N_{\mathrm{oc}}(M)$ goes to a constant value at large $M$. Here, we study this quantity in real space. The results of $N_{\mathrm{oc}}(M)$ are plotted against $M$ in the log-log scale in Fig. 2 where the RGF and TM are represented by triangles and circles, respectively. The overall agreement between two calculations is very good. However, there are also some discrepancies when $M$ is large. This is because the vector $\hat{\mathbf{z}}_{M}$ used in Eq. (10) is obtained after $M$ orthonomalization steps in Eq. (9). Numerical inaccuracy accumulates when $M$ becomes large. It should be mentioned that both curves give a slope 0.54 (dotted line), which is close to the slope 0.5 found previously in Ref. 6 in the space of propagating channels. The results of this very general case again demonstrate the validity of Eq. (10) in describing the statistics of layer occupation probability. 
In summary, we have shown explicitly that, in the GramSchmidt orthonormalization procedure of the TM, the vector nature of the orthonormalized vector that corresponds to the localization length exponent contains the statistical information about layer occupation probability. Therefore, by using the TM, we can obtain not only all the Lyapunov exponents, but also the stationary properties of the system. The conclusions reached here can be extended to other physical problems involving multimode conversion.

This work was supported in part by Hong Kong RGC Grant No. HKUST 686/96P.
${ }^{1}$ For example, see, A. Crisanti, G. Paladin, and A. Vulpiani, Products of Random Matrices in Statistical Physics (Springer-Verlag, Berlin, 1993).

${ }^{2}$ I. Goldhirsch, P. L. Sulem, and S. A. Orszag, Physica D 27, 311 (1987).

${ }^{3}$ A. MacKinnon and B. Kramer, Z. Phys. B 53, 1 (1983).

${ }^{4}$ A. D. Stone, P. A. Mello, K. A. Muttalib, and J. L. Pichard, in Mesocopic Phenomena in Solids, edited by B. L. Altshuler, P. A. Lee, and R. A. Webb (North-Holland, Amsterdam, 1991).

${ }^{5}$ W. Xue, P. Sheng, Q. J. Chu, and Z. Q. Zhang, Phys. Rev. Lett.
63, 2837 (1989); Z. Q. Zhang, Q. J. Chu, W. Xue, and P. Sheng, Phys. Rev. B 42, 4613 (1990).

${ }^{6}$ P. Sheng and Z. Q. Zhang, Phys. Rev. Lett. 74, 1343 (1995).

${ }^{7}$ Z. Q. Zhang and K. C. O, Phys. Rev. B 53, R11 917 (1996).

${ }^{8}$ Kh. M. Zakhar-Itkin, Radio Eng. Electron. Phys. 22, 1 (1977).

${ }^{9}$ W. Kohler, G. Papanicolaou, and B. White, Wave Motion 23, 1 (1996); 23, 181 (1996).

${ }^{10}$ P. A. Lee and D. Fisher, Phys. Rev. Lett. 47, 882 (1981).

${ }^{11}$ W. Apel and T. M. Rice, J. Phys. C 16, L1151 (1983); Q. Li, C. M. Soukoulis, E. N. Economou, and G. S. Grest, Phys. Rev. B 40, 2825 (1989). 